
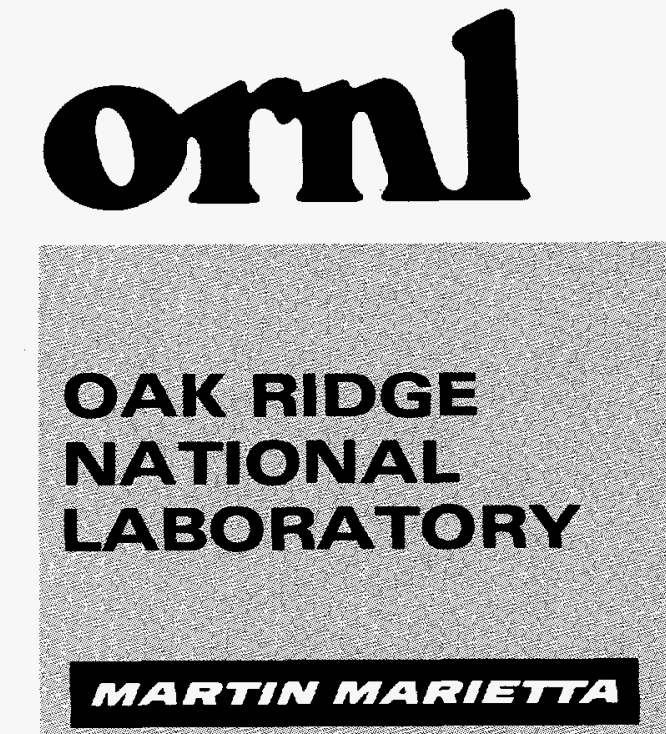

MAGTIN MARIETRA

MARTIN MARIETTA ENERGY SYSTEMS, INC. FOR THE UNITED STATES DEPARTMENT OF ENERGY

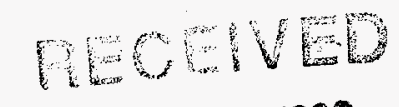

ORNL/M-13117

\title{
Terrain Coverage of an Unknown Room by an Autonomous Mobile Robot
}

Julie R. VanderHeide

Nageswara S. V. Rao 
This report has ben reproduced directly from the best available copy.

Available to DOE and DOE contractors from the Office of Scientific and Technical Information, P.O. Box 62, Oak Ridge, TN 37831; prices available from (615) 576-8401, FTS 626-8401.

Available to the public from the National Technical Information Service, U.S. Department of Commerce, 5285 Port Royal Rd., Springfield, VA 22161.

This report was prepared as an account of work sponsored by an agency of the United States Government. Neither the United States Government nor any agency thereof, nor any of their employees, makes any warranty, express or implied, or assumes any legal liability or responsibility for the accuracy. completeness, or usefulness of any information, apparatus, product, or process disclosed, or represents that its use would not infringe privately owned rights. Reference herein to any specific commercial product, process, or service by trade name, trademark, manufacturer, or otherwise, does not necessarily constitute or imply its endorsement, recommendation, or favoring by the United States Government or any agency thereot. The views and opinions of authors expressed herein do not necessarily state or reflect those of the United States Government or any agency thereot. 
ORNL/TM-13117

\title{
Terrain Coverage of an Unknown Room by an Autonomous Mobile Robot
}

\author{
Julie R. VanderHeide \\ Department of Electrical Engineering \\ Michigan State University \\ East Lansing,MI 48825 \\ Nageswara S. V. Rao
}

DATE PUBLISHED — 5 December 1995

Research sponsored by the
Science and Engineering Research Semester Program
and
Office of Basic Energy Sciences
U.S. Department of Energy

\author{
Prepared by the \\ OAK RIDGE NATIONAL LABORATORY \\ Oak Ridge, Tennessee 37831 \\ managed by \\ LOCKHEED MARTIN ENERGY RESEARCH CORP. \\ for the \\ U.S. DEPARTMENT OF ENERGY \\ under contract DE-AC05-96OR22464
}


.

, 


\section{Contents}

Acknowledgements $\quad$ v

Abstract vi vi vis

1 Introduction 1

2 Background 2

3 Terrain Coverage 2

4 Experimental Setup 5

5 Experimental Results $\quad 7$

6 Conclusion $\quad 8$ 


\section{List of Figures}

1 Flowchart of robot control. . . . . . . . . . . . . . 3

2 Room decomposed into rectangles. . . . . . . . . . . 4

3 Path planned for individual rectangles. . . . . . . . . . 4

4 TRC Labmate with sonar arrays. . . . . . . . . . . . . . . 6

5 Example of Labmate terrain coverage of an empty room. . . . . . . 7

6 Example of terrain coverage with an obstacle. . . . . . . . 8 


\section{Acknowledgements}

The support of this work by Oscar Manley of the Basic Energy Sciences Program in the U.S. Department of Energy is acknowledged. The first author was supported in part by the Science Engineering Research Semester Program of the U.S. Department of Energy. This research is also sponsored by the Oak National Laboratory, managed by Lockheed Martin Energy Research Corp. for the U.S. Department of Energy under contract number DE-AC05-96OR22464. 


\section{Abstract}

The problem of covering a terrain whose model is not a priori known by a mobile robot is considered here. The proposed solution consists of several subtasks: following the outer boundary of room(s), planning a path for terrain coverage, controlling the robot along the planned path, and adapting the path when obstacles are encountered. The wall-following module, path planning module, and path execution module have been designed and tested in rooms with rectilinear walls. The path adaptation module has been completed for the case of a single obstacle of arbitrary size and shape. The present implementation is based on a TRC Labmate mobile robot controlled from a remote SPARC workstation. The robot is equipped with an array of ultrasonic and binary infrared sensors. Potential applications of this system include radiation scanning, vacuum cleaning, autonomous model building, etc. 


\section{Introduction}

Terrain coverage problems are nearly as old as mankind: they were necessary early in our history for basic activities such as finding food and other necessities. As our societies and their associated machineries have grown more complex, we have not outgrown the need for this primitive skill. It is still used on a small scale for cleaning tasks and on a large scale for "search and report" missions of various kinds. The motivation for automating this process may not lie in the novelty of anything we might gain as an end product, but in freedom from something which we as humans find tedious, time-consuming and sometimes dangerous.

Here we consider autonomous coverage of a terrain, typically indoor rooms, by a mobile robot that has no a priori model of the terrain. In evaluating its surroundings, the robot employs only inexpensive and commercially available ultrasonic and infrared sensors. The proposed solution is a basic step - a proof of principle - that can contribute to robots capable of autonomously performing tasks such as vacuum cleaning, mopping, radiation scanning, etc.

The area of automatic terrain coverage and the closely related problem of terrain model acquisition have been studied both analytically and experimentally. Compared to the existing works, the following are three major distinguishing aspects of our study: 1) the theory is actually applied to an existing robot, 2) the robot has no a priori knowledge of the terrain, and 3 ) the robot can be realized relatively inexpensively.

Many papers have been, written on robot path planning and mobile robot navigation [2]. Computer scientists have shown that terrain model acquisition of certain types of areas is mathematically possible $[4,6]$; these techniques can be adapted to the terrain coverage problems. But the fact that this may be possible in theory does not necessarily lead to a practical, real-time and low-cost solution.

Other recent work has involved practical, real-time implementation of the terrain coverage task, but has required that a map of the area to be covered be programmed into the robot beforehand [1]. In contrast to these other projects, our project involves a robot starting at some location in an unknown room.

This project has been complicated by the desire to keep the robot low cost. In order to make possible the use of this robot as a widely available, practical cleaning tool, efforts have been made to keep the robot relatively inexpensive. This has translated into a robot in which position and heading errors can accumulate quite rapidly. It has also meant limiting the sensors to low cost ultrasonic and binary infrared, two highly error-prone sensor types, though it is unclear whether more expensive sensors would alleviate the technical difficulties.

Much in today's popular media might indicate that autonomous terrain coverage is trivial or can already be accomplished by existing, low-cost and commercially available

robots. Nothing could be further from the truth. As stated earlier, the automation of terrain coverage would be very useful both for industries and private homes. If such robots were seen as possible by industry, the robots would have already been mass produced at great profit to the holder of the patent.

Section 3 will describe an algorithm for terrain coverage. Section 4 will then cover 
the conditions under which this technique was developed and implemented and will be followed by experimental results in Section 5 and conclusions in Section 6 .

\section{Background}

The first important distinction that must be made is between path planning and terrain coverage. Path planning involves computing a path to move a robot from point A to point B [2]. The actual path taken is of no great import except for optimization. Terrain coverage on a lower level, in contrast to path planning, is concerned with little other than the path taken. The goal of terrain coverage is to pass over as much floor surface area as is possible, much as one would do when vacuuming. The many papers written about path planning [3] should not cause one to believe that the problem of terrain coverage has already been solved.

Another closely related problem is terrain model acquisition [5], where an automonous robot is required to completely build a model of the terrain. Despite the apparent similarity, these two problems have different objectives. Terrain coverage requires the robot to cover all free space within the bounded area, i.e. to go over all of the floor area not obscured by obstacles. Terrain acquisition, on the other hand, requires that the obstacles be modelled. In terms of so called non-heuristic algorithms we are unaware of any terrain coverage algorithms that have been proven to converge. The methods of terrain model acquisition can, however, be adapted to achieve terrain coverage.

In terms of practical systems a very important point which distinguishes this project from others is that this project deals with an unknown terrain. Other projects have been done using a model of the room preprogrammed into the robot's memory. Although the robot may be capable of autonomous terrain coverage, it requires a skilled human operator to enter data for each new room it comes across [1]. In contrast, the technique used in this project requires only that the robot be left in a room somewhat near a wall and away from obstacles and that a start button be pushed. The robot will learn the necessary parameters of the room on its own accord.

A final point is in reference to the practicality of the technique. By adapting the methods for terrain model acquisition, in particular using trapezoidal decomposition [4], terrain coverage can be shown in a formal framework to be attainable, yet this usually rests on the assumption of an ideal robot with ideal sensors. This project involved not only the development, but also the implementation of the technique about to be described.

\section{Terrain Coverage}

The following assumptions and /or limitations were placed on this project:

1. The room must have straight walls and square corners. The robot uses the walls of the room to correct heading errors.

2. Only one obstacle can be present in a given room. 


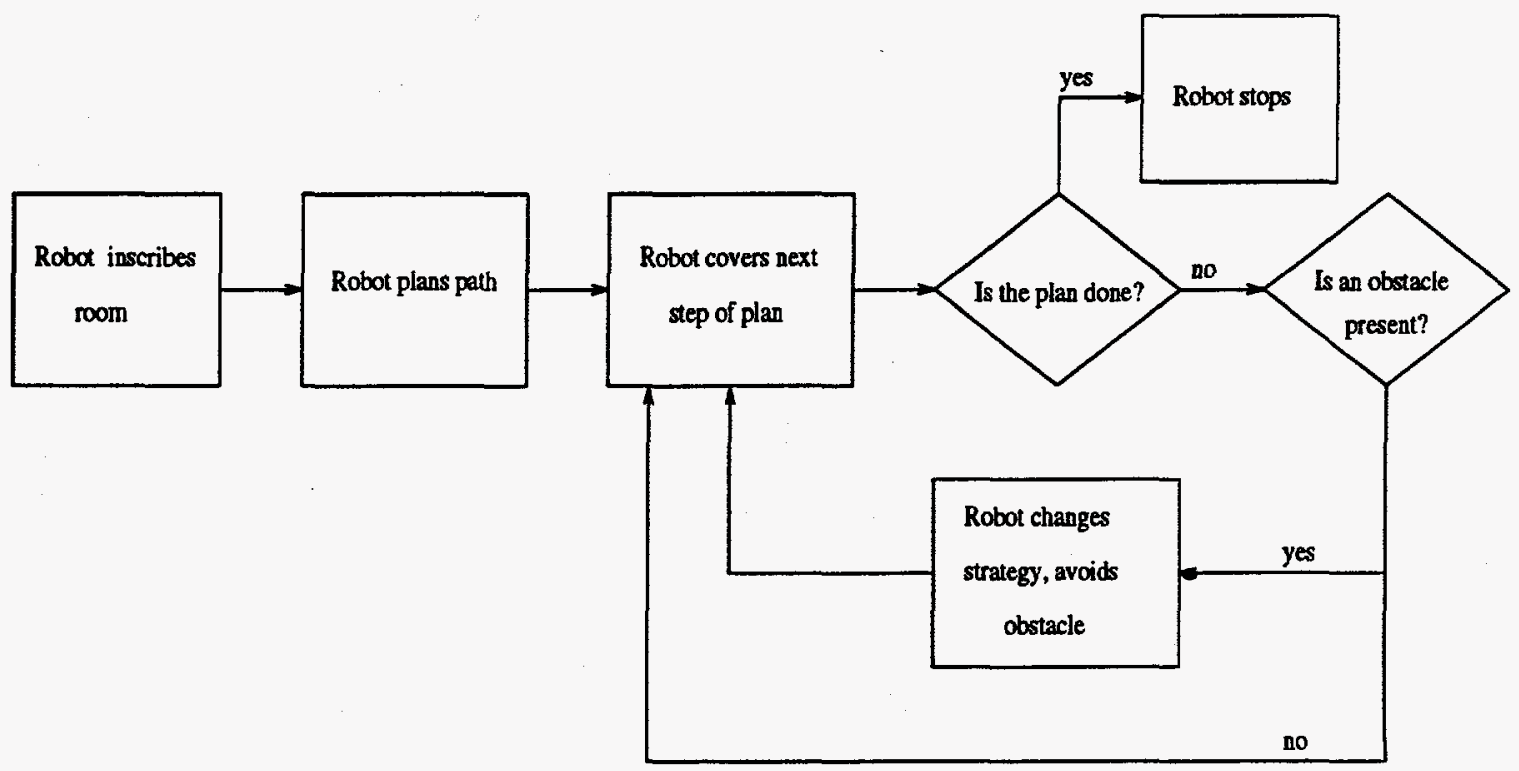

Figure 1: Flowchart of robot control.

3. If an obstacle is in the room the operator must place the robot near a wall and away from any obstacles to start it.

4. The obstacle must be large enough on each side that it can be sensed by the robot. For typical wood, metal, or hard plastic this means at least one to two inches in each dimension.

5. The obstacle must be stationary.

The process we developed for autonomous terrain coverage consists of five stages. The last two are optional.

1. The first stage consists of the robot inscribing the room and reporting what it finds. This gives the program a preliminary sketch of the room.

2. In stage two the information obtained in stage one is used to plan a path for the room.

3. The third stage is to actually cover the terrain, making adjustments for any obstacles encountered.

4. The fourth stage takes the robot back to the obstacle.

5. In the fifth stage the robot circumscribes the last obstacle encountered.

A more detailed description of each stage follows.

Starting wherever the operator left it, the robot first finds a concave corner to start in. From there it follows the wall around the room. A simple bang-bang control algorithm based on ultrasonic data is used to follow the wall. The length of each wall and direction of each turn are stored. This completes stage one. 


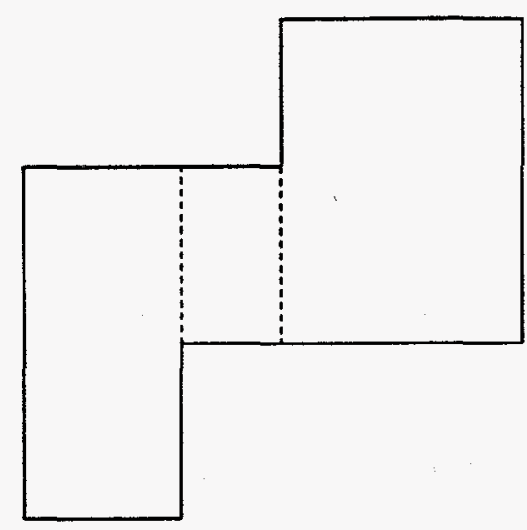

Figure 2: Room decomposed into rectangles.

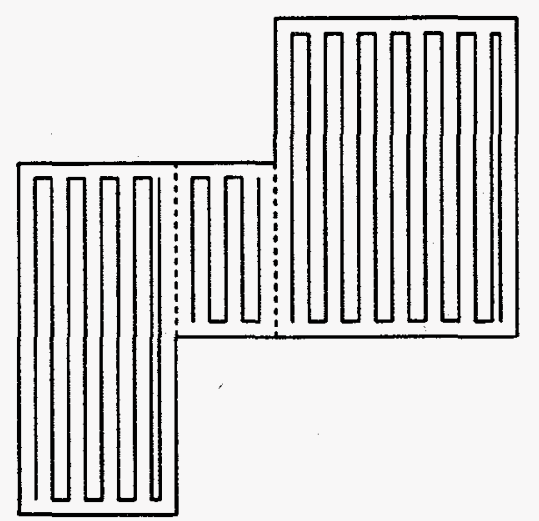

Figure 3: Path planned for individual rectangles.

Stage two is the planning algorithm. This module reads in the values stored in part one. It views the data, i.e. the parameters of the room, as a series of vertical and horizontal lines and decomposes the room into a set of rectangles. Figure 2 is an illustrative example of this. Rectangles are opened, closed or both for each vertical line. This produces a number of rectangles together representing the room. A depthfirst-search is performed on the rectangles to obtain the order in which they will be covered by the robot. A terrain coverage plan is then generated for each rectangle; an example of this is shown in Figure 3. Finally, the plans for each rectangle are strung together into a single path for the robot to follow. Additional plans are made which would take the robot from one end of each rectangle to the other in the event that an obstacle blocks the full width of the given rectangle. The essential parts of this plan, including length of each rectangle, number of times the robot needs to traverse each rectangle, and plans for getting from one rectangle to another, are stored for part three.

Stage three returns to robot control. The robot covers the path computed in stage two. Sensory data is used to correct for the robot's errors in position, heading, and turn angles. At the end of each lap of traversal in a rectangle the robot uses sensory information to correct its alignment with respect to the walls. The robot watches for obstacles on its sides as it traverses the room. The robot also knows that it has 
encountered an obstacle on the front if its last traversal of the room was not long enough. If an obstacle is encountered, the robot will switch to a separate module for obstacles. The robot will iteratively go from the wall to the obstacle and back until it finds the end of a rectangle or the end of the obstacle. The robot covers the terrain on a rectangle-by-rectangle basis regardless of obstacles. This module enables the robot to avoid the obstacle while still covering all of the area possible.

Stage four takes the robot back to the last obstacle in two turns and two lengths.

Circumscribing the obstacle is the job of the last stage. The robot follows around the obstacle, the end of the path overlapping the beginning. This stage is meant to cover area that the first terrain coverage did not. This stage is not advisable when the obstacle comes within one and a half robot widths of the wall and is not possible when the shape of the room is other than rectangular.

\section{Experimental Setup}

The experimental robot consists of a mobile platform, Labmate, commercially produced by Transitions Research Corp. (TRC) and a sensor configuration built by the second author. The platform and proximity subsystem are controlled by a SPARCstation $1+$ via a wireless radio modem. The position and sensor information is displayed using a simple software system based on Xlib.

The mobile platform has minimal processing abilities. It holds values for the current position, heading, wheel velocities and other strategic variables. It is powered by two on-board rechargeable 12 volt batteries. Because the Labmate has its power supply on-board and is controlled by a wireless modem it is untethered, i.e. it doesn't have any cords hanging out. The Labmate is capable of translational and rotational motion in a "car-like" fashion.

The proximity subsystem consists of 16 binary infrared and 16 ultrasound sensors. Each infrared sensor sends out a beam of infrared light. The sensor returns a true signal or a false signal depending on the intensity of any reflected beam detected. This true or false signal is the interpreted as the presence or absence of an obstacle. A schematic of the sensor-system is shown in Figure 4. Notice that the ultrasonic sensors have limited resolution due to a scan "cone" and the infrared sensors are highly directional. Further, the readings of ultrasonic sensors is affected by phenomenon such as specular and multiple reflections and the infrared sensors are affected by surface color, texture, etc.

The mobile platform and proximity subsystem are tied together by a logic board supplied by TRC. This board may be thought of as a "timing board" queuing commands to the robot and messages back to the computer, allowing only one member to "speak" at any given time.

Data returned by the robot are used as control signals thereby allowing the SPARCstation to maintain closed loop control over the robot. 
I'op-view of IRC Labmate
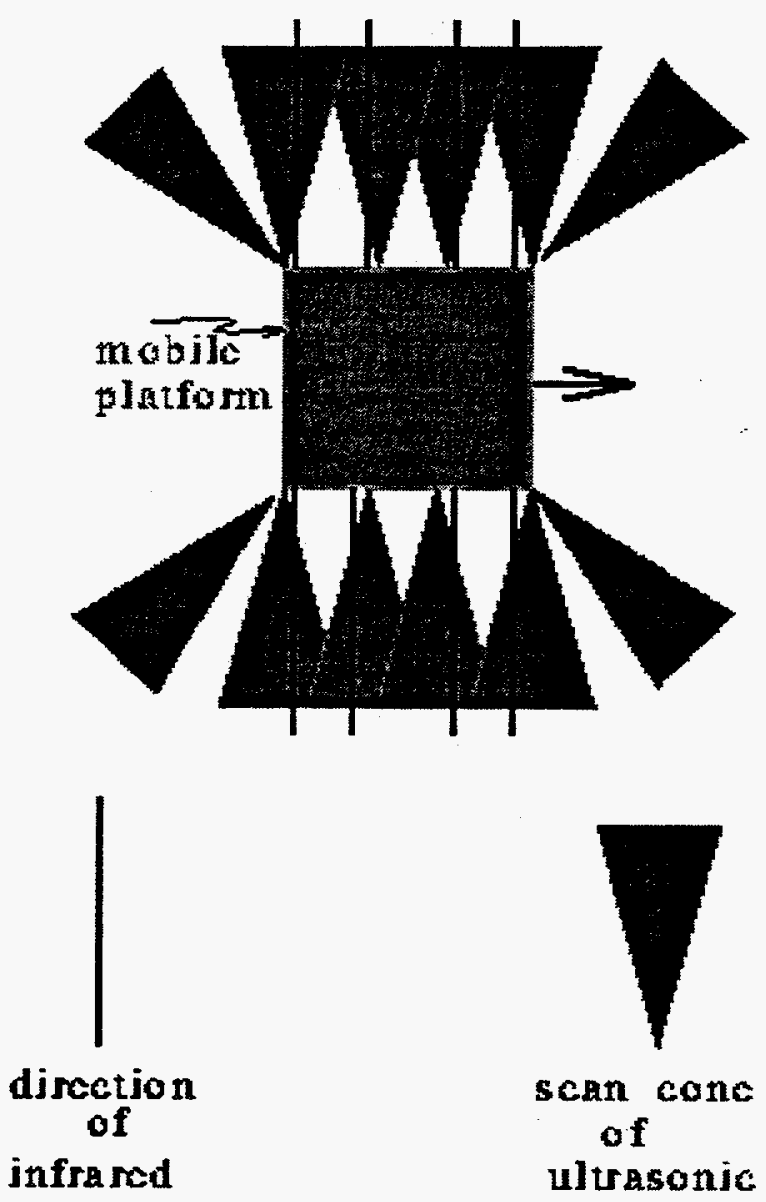

Figure 4: TRC Labmate with sonar arrays. 


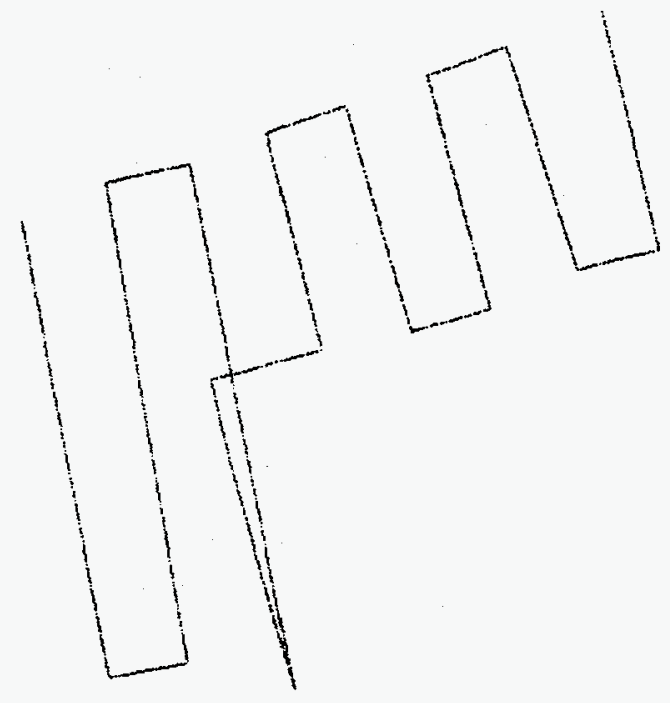

Figure 5: Example of Labmate terrain coverage of an empty room.

\section{Experimental Results}

\section{Empty Room}

Terrain coverage of an empty room works well. The lengths reported in stage one need not add up to a closed room. The planning function will change the lengths of the first two and/or last two segments in order to close the room. In practice, we often had discrepancies of $400 \mathrm{~mm}$ or more. A typical run is shown in Figure 5 for an L-shaped room; the figure shows the odometry readings corresponding to a complete coverage of the room. The lengths of the longest sides of this room are $520 \mathrm{~mm}$ and $610 \mathrm{~mm}$. The first stage of this run took about 6 minutes; terrain coverage itself took about 8 minutes.

\section{Room with Obstacles}

As arranged in Figure 4, the sensors are all about half of a meter off the ground. This causes problems with visibility of obstacles. Running tests with up to six ultrasound sensors on a side has not caused any problematic interference. We therefore propose two to four additional sensors placed on the front and on the right and left sides making shorter obstacles and those with diagonal sides visible. Typical results are shown in Figure 6. The obstacle in this case consists of a table and a chair oriented at a small angle to the room. This room was about $460 \mathrm{~mm}$ by $610 \mathrm{~mm}$. The first stage took about 5 minutes to run; terrain coverage took about 20 minutes. The time taken by other stages was negligible. 


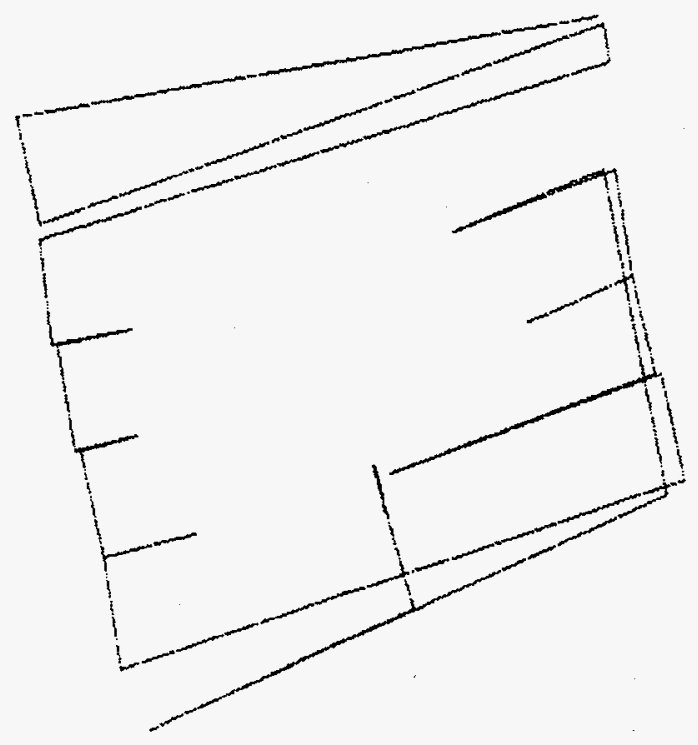

Figure 6: Example of terrain coverage with an obstacle.

\section{Conclusion}

In conclusion, a large step has been taken towards autonomous terrain coverage with a mobile robot. In this paper we have shown that (i) given a room with rectilinear walls and square corners, an autonomous mobile robot can ascertain its surroundings to the degree necessary for terrain coverage; (ii) a practical planning algoritm can be automated, and that terrain coverage of such a room with no more than one obstacle is attainable. While this is a large step forward, it is only a step. If such systems are ever to come into wide-spread usage, more work needs to be done. For large rooms robot alignment with the wall must be made more precise. Work needs to be done so that the robot can remember and avoid obstacles which are not completely visible. Perhaps the largest task which yet remains is to have the robot cover the terrain in multi-obstacle environments.

\section{References}

[1] C. Hofner and G. Schmidt. Path planning an guidance techniques for an autonomous mobile cleaning robot. Robotics and Autonomous Systems, 14:199-212, 1995.

[2] J. C. Latombe. Robot Motion Planning. Kluwer Academic Pub., Boston, 1991.

[3] H. S. Lin, J. Xiao, and Z. Michalewicz. Evolutionary algorithm for path planning in mobile robot environment. Proceedings of the 1st IEEE Conference on Evolutionary Computation. Part 1, 1:211-216, 1994.

[4] N. S. V. Rao. Robot navigation in unknown generalized polygonal terrains using vision sensors. IEEE Transactions on Systems, Man and Cybernetics, 25(6):947-962, 1995. 
[5] N. S. V. Rao, S. S. Iyengar, B. J. Oomen, and R. L. Kashyap. On terrain model acquisition by a point robot amidst polyhedral obstacles. IEEE Journal of Robotics and Automation, 3:450-455, 1988.

[6] N. S. V. Rao, S. Kareti, W. Shi, and S. S. Iyengar. Robot navigation in unknown terrains: An introductory survey of non-heuristic algorithms. Technical Report ORNL/TM12410, Oak Ridge National Laboratory, Oak Ridge, TN, 1993. 
ORNL/TM-13117

\section{INTERNAL DISTRIBUTION}
1. B. R. Appleton
2. M. Beckerman
3. C. W. Glover
4. J. P. Jones
5. R. C. Mann
6. E. M. Oblow
7. L. E. Parker
8-11. N. S. V. Rao

12. D. B. Reister

13. R. F. Sincovec

14. M. A. Unseren

15-16. Laboratory Records

17. Laboratory Records - RC

18. ORNL Patent Office

19. Central Research Library

20. CSMD Reports Office

\section{EXTERNAL DISTRIBUTION}

21. Dr. Oscar P. Manley, Division of Engineering, Mathematical and Geosciences, Office of Basic Energy Sciences, ER-15, U.S. Department of Energy - Germantown, Washington, DC 20545

22.-32 Julie R. VanderHeide, 4585 Jacob, Grandville, MI 49418

33. Dr. Mohammad Aslam, 260 Engineering Building, Michigan State University, East Lansing, MI 48825

34. Dr. Bong Ho, 260 Engineering Building, Michigan State University, East Lansing, MI 48825

35. Dr. Elias G. Strangas, 260 Engineering Building, Michigan State University, East Lansing, MI 48825

36. Office of the Assistant Manager for Energy Research and Development, Oak Ridge Operations, Department of Energy, P. O. Box 2008, Oak Ridge, TN 37831

37-38. Office of Scientific and Technical Information, Department of Energy, P. O. Box 62, Oak Ridge, TN 37831 


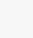

(1)
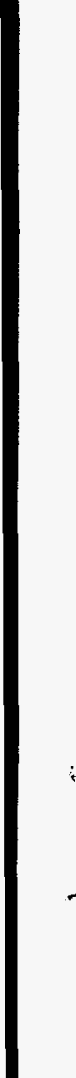
$L$

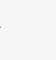

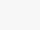

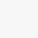

$\therefore$

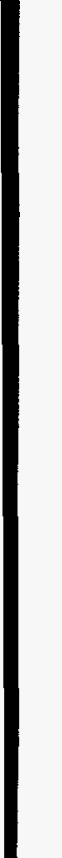

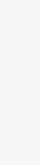
-

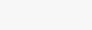


, 